Tôhoku Math. Journ.

29 (1977), 449-461.

\title{
BOUNDARY VALUE PROBLEMS FOR ORDINARY DIFFERENTIAL EQUATIONS
}

\author{
TAKASHI KAMINOGO
}

(Received May 24, 1976)

1. Introduction. We consider the boundary value problem for the second order scalar differential equation

$$
\begin{gathered}
x^{\prime \prime}=f\left(t, x, x^{\prime}\right) \\
x(a)=x_{a}, \quad x(b)=x_{b} .
\end{gathered}
$$

Hukuhara [1] proved the following Nagumo's existence theorem by using Kneser's property which will be stated in Section 2.

THEOREM 1.1. (Nagumo) Let $f(t, x, y)$ be a continuous function on $a$ compact domain $D: a \leqq t \leqq b, \underline{\omega}(t) \leqq x \leqq \bar{\omega}(t), \underline{\Omega}(t, x) \leqq y \leqq \bar{\Omega}(t, x)$, where $\underline{\omega}$ and $\bar{\omega}$ are twice continuously differentiable functions satisfying $\underline{\omega}(\bar{t}) \leqq \bar{\omega}(t)$ on $a \leqq t \leqq b, \underline{\Omega}$ and $\bar{\Omega}$ are continuously differentiable functions satisfying $\underline{\Omega}(t, x) \leqq \bar{\Omega}(t, x)$ on the domain $a \leqq t \leqq b, \underline{\omega}(t) \leqq$ $x \leqq \bar{\omega}(t)$. Suppose the following inequalities hold;

$$
\begin{aligned}
& \begin{cases}\underline{\Omega}(t, \underline{\omega}(t)) \leqq \underline{\omega}^{\prime}(t) \leqq \bar{\Omega}(t, \underline{\omega}(t)) & \text { for } \quad a \leqq t \leqq b \\
\underline{\Omega}(t, \bar{\omega}(t)) \leqq \bar{\omega}^{\prime}(t) \leqq \bar{\Omega}(t, \bar{\omega}(t)) & \text { for } \quad a \leqq t \leqq b,\end{cases} \\
& \begin{cases}\underline{\omega}^{\prime \prime}(t) \geqq f\left(t, \underline{\omega}(t), \underline{\omega}^{\prime}(t)\right) & \text { for } \quad a \leqq t \leqq b \\
\bar{\omega}^{\prime \prime}(t) \leqq f\left(t, \bar{\omega}(t), \bar{\omega}^{\prime}(t)\right) & \text { for } \quad a \leqq t \leqq b,\end{cases} \\
& \left\{\begin{array}{l}
f(t, x, \underline{\Omega}(t, x))-\underline{\Omega}_{t}(t, x)-\underline{\Omega}_{x}(t, x) \underline{\Omega}(t, x)>0 \\
f(t, x, \bar{\Omega}(t, x))-\bar{\Omega}_{t}(t, x)-\bar{\Omega}_{x}(t, x) \bar{\Omega}(t, x)<0
\end{array}\right. \\
& \text { for } a \leqq t \leqq b, \quad \underline{\omega}(t) \leqq x \leqq \bar{\omega}(t)
\end{aligned}
$$

and

$$
\underline{\omega}(a)=x_{a}=\bar{\omega}(a), \quad \underline{\omega}(b) \leqq x_{b} \leqq \bar{\omega}(b) .
$$

Then equation (1.1) has at least one solution which satisfies boundary condition (1.2).

Under conditions (1.4) and (1.5), inequalities (1.3) are essentially

$$
\begin{cases}\underline{\Omega}(t, \underline{\omega}(t))<\underline{\omega}^{\prime}(t) \leqq \bar{\Omega}(t, \underline{\omega}(t)) \text { for } \quad a<t \leqq b \\ \underline{\Omega}(t, \bar{\omega}(t)) \leqq \bar{\omega}^{\prime}(t)<\bar{\Omega}(t, \bar{\omega}(t)) \text { for } \quad a<t \leqq b .\end{cases}
$$


In Theorem 3.1 in this paper, assumptions (1.3), (1.4) and (1.5) of Theorem 1.1 will be weakened and furthermore, without the assumption $\underline{\omega}(a)=\bar{\omega}(a)$, we shall obtain solution $x(t)$ of (1.1) such that $\underline{\omega}(a) \leqq x(a) \leqq$ $\bar{\omega}(a)$ and $x(b)=x_{b}$.

\section{Kneser Family.}

Definition 2.1. A family $\mathscr{F}$ of $n$-vector valued continuous functions is called a family of curves if the following conditions are satisfied:

(a) Each curve $x$ (or $\left.\left\{(t, x(t)): t \in I_{x}\right\}\right)$ of $\mathscr{F}$ is a graph of an $n$ vector valued continuous function defined on a compact definition interval $I_{x}$.

(b) If $x$ belongs to $\mathscr{F}$, every partial arc $\left.x\right|_{I}$ (the restriction of $x$ to a compact subinterval $I$ of $I_{x}$ ) belongs to $\mathscr{F}$.

(c) $\mathscr{F}$ is a compact set in a metric space of compact sets in $R^{n+1}$, where the distance is defined by

$$
\begin{gathered}
\operatorname{Dist}(A, B)=\inf \left\{\delta>0: U_{\delta}(A) \supset B, \quad U_{\delta}(B) \supset A\right\}, \\
U_{\delta}(A)=\left\{p \in R^{n+1}: \operatorname{dist}(p, A) \leqq \delta\right\} .
\end{gathered}
$$

(d) If $x$ and $y$ of $\mathscr{F}$ assume a same value at $t=\alpha$, the function which coincides with $x$ for $t \leqq \alpha$ and with $y$ for $t \geqq \alpha$ belongs to $\mathscr{F}$.

(e) The end points of a maximal (with respect to the definition interval) curve belong to the boundary $B=\partial D$ of $D$, where $D=D(\mathscr{F})$ is the compact set in $R^{n+1}$ filled by the curves of $\mathscr{F}$ and is called the fundamental domain of $\mathscr{F}$.

Let $\mathscr{F}$ be a family of curves. The left end point of a maximal curve of $\mathscr{F}$ will be called a left extreme point of $D$. All left extreme points of $D$ form a set, which we call the left boundary and denote by $B^{l}=B^{l}(\mathscr{F})$. We define the left emission zone $Z^{-}(E), E \subset D$, by $Z^{-}(E)=$ $\left\{(t, x(t)): x \in \mathscr{F}\right.$ for which there exists a $t_{0} \in I_{x}$ such that $\left(t_{0}, x\left(t_{0}\right)\right) \in E$, $\left.t \leqq t_{0}\right\}$. The set $Z^{-}(\{p\})$ is simply denoted by $Z^{-}(p)$. The set of points $p \in B \backslash B^{l}$ such that $p$ is an isolated point of $B \cap Z^{-}(p)$ will be denoted by $B^{-}=B^{-}(\mathscr{F})$. The set of points $p \in B \backslash B^{l}$ such that $p$ is an accumulation point of $B \cap Z^{-}(p)$ will be denoted by $B_{-}=B_{-}(\mathscr{F})$. Then $B$ is expressed as the disjoint sum of $B^{l}, B^{-}$and $B_{-}$. Similarly, we define the right boundary $B^{r}$ and the set $B^{+}, B_{+}$.

Definition 2.2. Let $\mathscr{F}$ be a family of curves. A point $p=(\alpha, \xi) \in$ $D \subset R \times R^{n}$ is called a left Kneser point if it satisfies one of the following conditions:

( I ) $p$ is a point of $B^{l}$. 
(I I) $p$ is a point of $B^{-} \cup \operatorname{Int} D$ and the intersection of the emission zone $Z^{-}(p)$ with the hyperplane $t=\tau$ is a continuum when $\alpha-\tau>0$ is sufficiently small.

(III) $p$ is a point of $B_{-}$and the union $S \cup\left(B \cap Z_{\tau}^{-}(p)\right)$ is a continuum when $\alpha-\tau>0$ is sufficiently small, where

$$
\begin{aligned}
S & =\left\{(t, \zeta) \in Z^{-}(p): t=\tau\right\}, \\
Z_{\tau}^{-}(p) & =\left\{(t, \zeta) \in Z^{-}(p): t \geqq \tau\right\} .
\end{aligned}
$$

Definition 2.3. A family of curves $\mathscr{F}$ is called a left Kneser family if it satisfies the following condition:

(f) All points of $D$ are left Kneser points, and $B^{-}$is open in $B$ and is contained in $B^{r}$.

Hukuhara proved the following results .

THEOREM 2.1. If $\mathscr{F}$ is a left Kneser family, then the intersection

$$
Z^{-}(E) \cap\left(B^{l} \cup B_{-}\right)
$$

is a continuum when $E$ is a continuum in $D$.

For the proof, see [1].

LEMMA 2.1. Let $\mathscr{G}$ be a family of curves and $G$ be the fundamental domain of $\mathscr{G}$, and $\mathscr{F}$ be the family of the curves of $\mathscr{G}$ which are contained in a compact set $D$ in $G$. (It is easy to show that $\mathscr{F}$ is a family of curves and $D$ is the fundamental domain of $\mathscr{F}$.) Suppose that a point $p=(\alpha, \xi)$ of $B_{-}(\mathscr{F})$ is an interior point of $G$ or a point of $B^{-}(\mathscr{G})$, and that the following conditions are satisfied:

(i) Every maximal function (or curve) of $\mathscr{G}$ issuing from $p$ to the left exists uniformly in a sufficiently small interval $[\alpha-\delta, \alpha]$.

(ii) Every point of $Z_{\alpha-\delta}^{-}(p ; \mathscr{G})$ is a left Kneser point with respect to $\mathscr{G}$.

(iii) Any curve of $\mathscr{G}_{\alpha-\dot{\delta}}^{-}(p)$ issuing from an exterior point of $D$ to the left.cannot attain $D$, where $\mathscr{G}_{\alpha-\delta}^{-}(p)$ is the family of the curves of $\mathscr{G}$ which are contained in $Z_{\alpha-\dot{0}}^{-}(p ; \mathscr{G})$.

(iv) Any point of $B^{-}(\mathscr{F})$ does not belong to $Z_{\alpha-\hat{o}}^{-}(p ; \mathscr{F})$. Then $p$ is a left Kneser point of $\mathscr{F}$.

For the proof, see [1].

3. Existence Theorem. First we consider the system

$$
x^{\prime}=\varphi(t, x),
$$

where $\varphi$ is continuous on $X=[a, b] \times R^{n},-\infty<a<b<\infty$. 
Definition 3.1. A set $N$ in $X$ is a negatively invariant set with respect to (3.1) if for each point $\left(t_{0}, x_{0}\right) \in N$ and each solution $x(t)$ of (3.1) with $x\left(t_{0}\right)=x_{n},(t, x(t)) \in N$ on $\left[a, t_{0}\right] \cap \widetilde{J}_{x}$, where $\widetilde{J}_{x}$ is the maximal interval of existence of $x$. Similarly, we define a positively invariant set with respect to (3.1).

Lemma 3.1. Let $D$ be a compact subset of $X$ and $p=(\alpha, \xi)$ be a point of $D$. Let $\mathscr{F}$ be a family of solutions of (3.1) which are contained in $D$ and defined on compact intervals. Obviously $\mathscr{F}$ is a family of curves. Suppose that there is a negatively invariant set $N$ and a positively invariant set $P$ of (3.1) such that $X \backslash D=N \cup P$. Then (a) if $p$ is a point of $\bar{N}$ (the closure of $N$ ), $p$ is a point of $B^{l} \cup B_{-}$.

(b) if $B^{-} \subset B^{r}, p$ is a left Kneser point.

Proof. (a) Obviously, $p$ is a point of $B=\partial D$ since $p$ is a point of $\bar{N} \cap D$. Assume $p$ is not a point of $B^{l}$. Then there is a solution $y(t)$ of (3.1) issuing from $p$ to the left and a number $\sigma, \sigma<\alpha$, such that

$$
(t, y(t)) \in D \quad \text { on } \quad[\sigma, \alpha] .
$$

Let $\left\{p_{n}\right\}$ be a sequence in $N$ which converges to $p$ and $x_{n}$ be a left maximal solution of (3.1) issuing from $p_{n}$ to the left defined on $J_{n}$. Since $N$ is negatively invariant, we have

$$
\left(t, x_{n}(t)\right) \in N \quad \text { on } J_{n} \text {. }
$$

By Theorem 3.2 ([2], p. 14), there is a left maximal solution $x(t)$ of (3.1) issuing from $p$ to the left defined on $J_{x}$ and a subsequence of $\left\{x_{n}\right\}$ which converges to $x$ uniformly on any compact interval in $J_{x}$. Therefore we have

$$
(t, x(t)) \in \bar{N} \quad \text { on } \quad J_{x} .
$$

It then follows from (3.2), (3.3) and Kneser's theorem ([2], p. 15) that if $\tau$ in $J_{x} \cap[\sigma, \alpha)$ is sufficiently near to $\alpha$, there exists a solution $z(t)$ of (3.1) issuing from $p$ to the left defined on $[\tau, \alpha]$ such that

$$
(\tau, z(\tau)) \in B \text {. }
$$

Now we shall show that $(t, z(t)) \in D$ on $[\tau, \alpha]$. Suppose there is a $t_{1}, \tau<t_{1}<\alpha$, such that $\left(t_{1}, z\left(t_{1}\right)\right) \notin D$. If the point $\left(t_{1}, z\left(t_{1}\right)\right)$ is in $N$, then $(\tau, z(\tau))$ is in $N$ since $N$ is a negatively invariant set of (3.1). If the point $\left(t_{1}, z\left(t_{1}\right)\right)$ is in $P$, then $p$ is in $P$ since $P$ is a positively invariant set of (3.1). In both cases, there arise con tradictions. This shows $(t, z(t)) \in D$ on $[\tau, \alpha]$. Since the number $\tau$ can be assumed to be arbitrarily near to $\alpha$, we have $p \in B_{-}$. 
(b) If $p$ is a point of $B^{l} \cup B^{-} \cup$ Int $D, p$ is a left Kneser point by Kneser's theorem. Assume $p$ is a point of $B_{-}=B_{-}(\mathscr{F})$. Let $G$ be a compact subset of $X$ such that the interior of $G$ in $X$ containes $D$, and $\mathscr{F}$ be the family of solutions of (3.1) contained in $G$ and defined on compact intervals. Then one can easily verify that conditions (i) and (ii) in Lemma 2.1 are satisfied. By the same arguments as in the last part of the proof of (a), condition (iii) in Lemma 2.1 is satisfied. The assumptions $B^{-}(\mathscr{F}) \subset B^{r}(\mathscr{F})$ and $p \in B_{-}(\mathscr{F})$ imply condition (iv) in Lemma 2.1. Thus, by Lemma 2.1, we can conclude that $p$ is a left Kneser point of $\mathscr{F}$.

q.e.d.

THEOREM 3.1. Let $f$ on $D, \underline{\omega}, \bar{\omega}, \underline{\Omega}$ and $\bar{\Omega}$ be the functions given in Theorem 1.1. Instead of conditions (1.3), (1.4) and (1.5), suppose that these functions satisfy the following inequalities:

$$
\begin{aligned}
& \begin{cases}\underline{\omega}^{\prime}(t) \geqq \underline{\Omega}(t, \underline{\omega}(t)) & \text { for } \quad a \leqq t \leqq b \\
\bar{\omega}^{\prime}(t) \leqq \bar{\Omega}(t, \bar{\omega}(t)) & \text { for } \quad a \leqq t \leqq b,\end{cases} \\
& \begin{cases}\underline{\omega}^{\prime \prime}(t) \geqq f\left(t, \underline{\omega}(t), \underline{\omega}^{\prime}(t)\right) & \text { if } \quad \underline{\omega}^{\prime}(t) \leqq \bar{\Omega}(t, \underline{\omega}(t)) \\
\bar{\omega}^{\prime \prime}(t) \leqq f\left(t, \bar{\omega}(t), \bar{\omega}^{\prime}(t)\right) & \text { if } \quad \bar{\omega}^{\prime}(t) \geqq \underline{\Omega}(t, \bar{\omega}(t)),\end{cases}
\end{aligned}
$$

and

$$
\begin{gathered}
\left\{\begin{array}{c}
f(t, x, \underline{\Omega}(t, x))-\underline{\Omega}_{t}(t, x)-\underline{\Omega}_{x}(t, x) \underline{\Omega}(t, x) \geqq 0 \\
f(t, x, \bar{\Omega}(t, x))-\bar{\Omega}_{t}(t, x)-\bar{\Omega}_{x}(t, x) \bar{\Omega}(t, x) \leqq 0
\end{array}\right. \\
\text { for } a \leqq t \leqq b, \quad \underline{\omega}(t) \leqq x \leqq \bar{\omega}(t)
\end{gathered}
$$

Then for any number $x_{b}, \underline{\omega}(b) \leqq x_{b} \leqq \bar{\omega}(b)$, equation (1.1) has at least one solution $x(t)$ defined on $[a, b]$ such that $x(b)=x_{b}$. If $\underline{\omega}(a)=x_{a}=$ $\bar{\omega}(a)$, this solution satisfies boundary condition (1.2).

Proof. We consider an equivalent system

$$
x^{\prime}=y, \quad y^{\prime}=f(t, x, y) \text {. }
$$

Let $\mathscr{F}$ be the family of solutions of (3.7) in $D$ defined on compact intervals. First we shall show that the family $\mathscr{F}$ is a left Kneser family. We investigate the properties of boundary points of $D$.

The points in $B=\partial D$ which are on the plane $t=a$ belong to $B^{l}$. We denote by $S_{0}$ the set of such points.

Let $S_{1}$ be the set of points $(t, x, y) \in B$ such that

$$
t=b, \quad \underline{\omega}(b)<x<\bar{\omega}(b), \quad \underline{\Omega}(b, x)<y<\bar{\Omega}(b, x)
$$

or

$$
t \in I_{0}, \quad x=\underline{\omega}(t), \quad \underline{\Omega}(t, x)<y<\min \left\{\underline{\omega}^{\prime}(t), \quad \bar{\Omega}(t, x)\right\}
$$


or

$$
t \in I_{0}, \quad x=\bar{\omega}(t), \quad \operatorname{Max}\left\{\bar{\omega}^{\prime}(t), \underline{\Omega}(t, x)\right\}<y<\bar{\Omega}(t, x),
$$

where

$$
I_{0}=\{t: a<t \leqq b, \underline{\omega}(t)<\bar{\omega}(t)\} .
$$

The points of $S_{1}$ belong to $B^{-} \cap B^{r}$ since $y<\underline{\omega}^{\prime}(t)$ for the second case and $y>\bar{\omega}^{\prime}(t)$ for the third case.

Let $S_{2}$ be the set of points $(t, x, y) \in B$ such that

$$
a<t \leqq b, \quad \underline{\omega}(t) \leqq x \leqq \bar{\omega}(t), \quad y=\bar{\Omega}(t, x)
$$

or

$$
a<t \leqq b, \quad x=\underline{\omega}(t), \quad \underline{\omega}^{\prime}(t) \leqq y<\bar{\Omega}(t, x),
$$

and $S_{3}$ be the set of points $(t, x, y) \in B$ such that

$$
a<t \leqq b, \quad \underline{\omega}(t) \leqq x \leqq \bar{\omega}(t), \quad y=\underline{\Omega}(t, x)
$$

or

$$
a<t \leqq b, \quad x=\bar{\omega}(t), \quad \underline{\Omega}(t, x)<y \leqq \bar{\omega}^{\prime}(t) .
$$

We shall extend $f$ to $X=[a, b] \times R^{2}$ in order to show that the points of $S_{2} \cup S_{3}$ belong to $B^{l} \cup B_{-}$. First we construct a continuous extension $g^{*}$ of $f$ defined on a domain $a \leqq t \leqq b, \underline{\omega}(t) \leqq x \leqq \bar{\omega}(t),|y|<\infty$, so that the follwoing inequalities hold:

$$
\begin{array}{ll} 
& \underline{\omega}^{\prime \prime}(t) \geqq g^{*}\left(t, \underline{\omega}(t), \underline{\omega}^{\prime}(t)\right) \quad \text { for } \quad a \leqq t \leqq b, \\
& \bar{\omega}^{\prime \prime}(t) \leqq g^{*}\left(t, \bar{\omega}(t), \bar{\omega}^{\prime}(t)\right) \quad \text { for } \quad a \leqq t \leqq b, \\
& g^{*}(t, x, y) \leqq f(t, x, \bar{\Omega}(t, x)) \\
\text { for } \quad a \leqq t \leqq b, \quad \underline{\omega}(t) \leqq x \leqq \bar{\omega}(t), \quad y \geqq \bar{\Omega}(t, x)
\end{array}
$$

and

$$
\begin{aligned}
& g^{*}(t, x, y) \geqq f(t, x, \underline{\Omega}(t, x)) \\
& \quad \text { for } a \leqq t \leqq b, \quad \underline{\omega}(t) \leqq x \leqq \bar{\omega}(t), \quad y \leqq \underline{\Omega}(t, x) .
\end{aligned}
$$

Set $g^{*}=f$ on $D$. For $y \geqq \bar{\Omega}(t, x), g^{*}$ is constructed in the following way. For $t \in I_{1}, x=\underline{\omega}(t)$ and $y=\underline{\omega}^{\prime}(t)$, we define $g^{*}$ by

$$
g^{*}(t, x, y)=\min \left\{\underline{\omega}^{\prime \prime}(t), f(t, x, \bar{\Omega}(t, x))\right\}
$$

where

$$
I_{1}=\left\{t: a \leqq t \leqq b, \quad \underline{\omega}^{\prime}(t)>\bar{\Omega}(t, \underline{\omega}(t))\right\} .
$$

Then (3.8) holds. For $t \in I_{1}, x=\underline{\omega}(t)$ and $\bar{\Omega}(t, x)<y<\underline{\omega}^{\prime}(t)$, we define 
$g^{*}$ by joining $f(t, x, \bar{\Omega}(t, x))$ and $g^{*}\left(t, x, \underline{\omega}^{\prime}(t)\right)$ linearly in $y$, that is,

$$
\begin{aligned}
g^{*}(t, x, y) & \\
& =\frac{\left(\underline{\omega}^{\prime}(t)-y\right) f(t, x, \bar{\Omega}(t, x))+(y-\bar{\Omega}(t, x)) g^{*}\left(t, x, \underline{\omega}^{\prime}(t)\right)}{\underline{\omega}^{\prime}(t)-\bar{\Omega}(t, x)} .
\end{aligned}
$$

For $a \leqq t \leqq b, x=\underline{\omega}(t)$ and $y>\operatorname{Max}\left\{\underline{\omega}^{\prime}(t), \bar{\Omega}(t, x)\right\}=\gamma(t)$, let $g^{*}$ be

$$
g^{*}(t, x, y)=g^{*}(t, x, \gamma(t)) \text {. }
$$

For $a \leqq t \leqq b, \underline{\omega}(t)<x \leqq \bar{\omega}(t)$ and $y>\bar{\Omega}(t, x), g^{*}$ is defined by

$$
\begin{gathered}
g^{*}(t, x, y)=f(t, x, \bar{\Omega}(t, x))-f(t, \underline{\omega}(t), \bar{\Omega}(t, \underline{\omega}(t))) \\
+g^{*}(t, \underline{\omega}(t), \bar{\Omega}(t, \underline{\omega}(t))+y-\bar{\Omega}(t, x)) .
\end{gathered}
$$

Then (3.10) holds. Similarly, we can construct $g^{*}$ for $y \leqq \underline{\Omega}(t, x)$ so that (3.9) and (3.11) are satisfied. Finally, we define a continuous extension $g$ of $f$ defined on $X$ by

$$
g(t, x, y)= \begin{cases}g^{*}(t, \bar{\omega}(t), y)+x-\bar{\omega}(t) & \text { if } x>\bar{\omega}(t) \\ g^{*}(t, x, y) & \text { if } \underline{\omega}(t) \leqq x \leqq \bar{\omega}(t) \\ g^{*}(t, \underline{\omega}(t), y)+x-\underline{\omega}(t) & \text { if } x<\underline{\omega}(t) .\end{cases}
$$

Instead of system (3.7), we now consider the system

$$
x^{\prime}=y, \quad y^{\prime}=g(t, x, y) .
$$

We divide the set $X \backslash D$ into the following sets;

$$
\begin{aligned}
& D_{1}=\{(t, x, y): a \leqq t \leqq b, \underline{\omega}(t) \leqq x \leqq \bar{\omega}(t), y>\bar{\Omega}(t, x)\}, \\
& D_{2}=\left\{(t, x, y): a \leqq t \leqq b, x<\underline{\omega}(t), y \geqq \underline{\omega}^{\prime}(t)\right\}, \\
& D_{3}=\left\{(t, x, y): a \leqq t \leqq b, x<\underline{\omega}(t), y \leqq \underline{\omega}^{\prime}(t)\right\}, \\
& D_{4}=\{(t, x, y): a \leqq t \leqq b, \underline{\omega}(t) \leqq x \leqq \bar{\omega}(t), y<\underline{\Omega}(t, x)\}, \\
& D_{5}=\left\{(t, x, y): a \leqq t \leqq b, x>\bar{\omega}(t), y \leqq \bar{\omega}^{\prime}(t)\right\}
\end{aligned}
$$

and

$$
D_{6}=\left\{(t, x, y): a \leqq t \leqq b, x>\bar{\omega}(t), y \geqq \bar{\omega}^{\prime}(t)\right\}
$$

If $x(t)$ is a solution of (3.12) such that $x\left(t_{0}\right)<\underline{\omega}\left(t_{0}\right)$ and $x^{\prime}\left(t_{0}\right)=\underline{\omega}^{\prime}\left(t_{0}\right)$, then we have

$$
\begin{aligned}
x^{\prime \prime}\left(t_{0}\right) & =g\left(t_{0}, x\left(t_{0}\right), x^{\prime}\left(t_{0}\right)\right) \\
& =g^{*}\left(t_{0}, \underline{\omega}\left(t_{0}\right), \underline{\omega}^{\prime}\left(t_{0}\right)\right)+x\left(t_{0}\right)-\underline{\omega}\left(t_{0}\right) \\
& <g^{*}\left(t_{0}, \underline{\omega}\left(t_{0}\right), \underline{\omega}^{\prime}\left(t_{0}\right)\right) \leqq \underline{\omega}^{\prime \prime}\left(t_{0}\right) .
\end{aligned}
$$

This implies that $D_{2}$ is a negatively invariant set and $D_{3}$ is a positively invariant set with respect to (3.12). Let $x(t)$ be a solution of (3.12) 
issuing from a point of $D_{1}$, that is, $\underline{\omega}\left(t_{0}\right) \leqq x\left(t_{0}\right) \leqq \bar{\omega}\left(t_{0}\right)$ and $x^{\prime}\left(t_{0}\right)>\bar{\Omega}\left(t_{0}\right.$, $\left.x\left(t_{0}\right)\right)$ for some $t_{0}$. Along this solution $x(t)$, let

$$
V(t)=\left(x^{\prime}(t)-\bar{\Omega}(t, x(t))\right) \exp \int_{t_{0}}^{t} \bar{\Omega}_{x}(s, x(s)) d s .
$$

Then, as long as $\underline{\omega}(t) \leqq x(t) \leqq \bar{\omega}(t)$ and $x^{\prime}(t) \geqq \bar{\Omega}(t, x(t))$, we have

$$
\begin{aligned}
V^{\prime}(t) & \exp \left[-\int_{t_{0}}^{t} \bar{\Omega}_{x}(s, x(s)) d s\right] \\
& =x^{\prime \prime}(t)-\bar{\Omega}_{t}(t, x(t))-\bar{\Omega}_{x}(t, x(t)) \bar{\Omega}(t, x(t)) \\
& =g^{*}\left(t, x(t), x^{\prime}(t)\right)-\bar{\Omega}_{t}(t, x(t))-\bar{\Omega}_{x}(t, x(t)) \bar{\Omega}(t, x(t)) \\
& \leqq f(t, x(t), \bar{\Omega}(t, x(t)))-\bar{\Omega}_{t}(t, x(t))-\bar{\Omega}_{x}(t, x(t)) \bar{\Omega}(t, x(t)) \\
& \leqq 0
\end{aligned}
$$

From this and $V\left(t_{0}\right)>0$, we have $x^{\prime}(t)>\bar{\Omega}(t, x(t))$ as long as $\underline{\omega}(t) \leqq x(t) \leqq$ $\bar{\omega}(t), t \leqq t_{0}$. When $t$ decreases from $t_{0}$, if this solution arc does not remain in $D_{1}$, then it enters $D_{2} \cup D_{3}$ because $\bar{\omega}^{\prime}(t) \leqq \bar{\Omega}(t, \bar{\omega}(t))$. Furthermore, $D_{3}$ is positively invariant and $D_{1} \cap D_{3}=\varnothing$, and hence this solution arc enters $D_{2}$. Therefore $D_{1} \cup D_{2}$ is a negatively invariant set of (3.12). Similarly, we can show that $D_{4} \cup D_{5}$ is a negatively invariant set and $D_{6}$ is a positively invariant set of (3.12). Thus $N=D_{1} \cup D_{2} \cup$ $D_{4} \cup D_{5}$ is negatively invariant and $P=D_{3} \cup D_{6}$ is positively invariant with respect to (3.12) and $X \backslash D=N \cup P$.

Since $S_{2} \cup S_{3}$ is contained in $\bar{N}$, it is contained in $B^{l} \cup B_{-}$by Lemma 3.1(a). Therefore $B^{-}$is just $S_{1}$, and this implies that $B^{-}$is contained in $B^{r}$. It then follows from Lemma $3.1(\mathrm{~b})$ that all points of $D$ are left Kneser points. Consequently, $\mathscr{F}$ is a left Kneser family since $B^{-}=S_{1}$ is open in $B$.

Now let,

$$
E=\left\{\left(b, x_{b}, y\right): \underline{Q}\left(b, x_{b}\right) \leqq y \leqq \bar{Q}\left(b, x_{b}\right)\right\}
$$

and

$$
K=Z^{-}(E) \cap\left(B^{l} \cup B_{-}\right)
$$

Then

$$
K=Z^{-}(E) \cap\left(S_{0} \cup S_{2} \cup S_{3}\right)
$$

and $K$ is a continuum by Theorem 2.1 because $E$ is a continuum in $D$.

Next we shall show that $K \cap S_{0}$ is nonempty. Assume $K \cap S_{0}=\varnothing$. Let $L$ be a set of points $(t, x, y) \in B$ such that 


$$
a \leqq t \leqq b, \quad x=\underline{\omega}(t), \quad y=\underline{\omega}^{\prime}(t)=\underline{Q}(t, x)
$$

or

$$
a \leqq t \leqq b, \quad x=\bar{\omega}(t), \quad y=\bar{\omega}^{\prime}(t)=\bar{\Omega}(t, x)
$$

or

$$
a \leqq t \leqq b, \quad x=\underline{\omega}(t)=\bar{\omega}(t), \quad y=\underline{\omega}^{\prime}(t)=\bar{\omega}^{\prime}(t)
$$

or

$$
a \leqq t \leqq b, \quad \underline{\omega}(t) \leqq x \leqq \bar{\omega}(t), \quad y=\underline{\Omega}(t, x)=\bar{\Omega}(t, x)
$$

Then the intersection $M=K \cap L$ is a nonempty compact set because $K \cap S_{2} \neq \varnothing$ and $K \cap S_{3} \neq \varnothing$. Let $q$ be one of the left end points of $M$. We may assume $q$ is a point of the first case (i) since the arguments for the other cases are similar. Therefore we can write $q=$ $\left(\tau, \underline{\omega}(\tau), \underline{\omega}^{\prime}(\tau)\right)$ for some $\tau, a<\tau \leqq b$. By Theorem 2.1, the set $H=$ $Z^{-}(q) \cap\left(B^{l} \cup B_{-}\right)$is a continuum and is contained in $K$. Since $q \in \bar{D}_{2}$ and $D_{2}$ is a negatively invariant set of (3.12), there is a left maximal solution $y(t)$ of (3.12) issuing from $q$ to the left defined on $J_{y}$ such that

$$
\left(t, y(t), y^{\prime}(t)\right) \in \bar{D}_{2} \quad \text { on } \quad J_{y} \text {. }
$$

Similarly, there is a left maximal solution $z(t)$ of (3.12) issuing from $q$ to the left defined on $J_{z}$ such that

$$
\left(t, z(t), z^{\prime}(t)\right) \in \overline{D_{4} \cup D_{5}} \quad \text { on } \quad J_{z} \text {. }
$$

Since the set $D_{3}$ is positively invariant and does not contain $q$, any solution of (3.12) issuing from $q$ to the left cannot enter $D_{3}$. It follows from this and Kneser's theorem that there are two solutions $y_{0}(t)$ and $z_{0}(t)$ of (3.12) issuing from $q$ to the left and a number $\sigma, \sigma<\tau$, such that

$$
q_{y}=\left(\sigma, y_{0}(\sigma), y_{0}^{\prime}(\sigma)\right) \in S_{2}
$$

and

$$
q_{z}=\left(\sigma, z_{0}(\sigma), z_{0}^{\prime}(\sigma)\right) \in S_{3}
$$

Furthermore, we can show that

$$
\left(t, y_{0}(t), y_{0}^{\prime}(t)\right) \in D \quad \text { for } \quad \sigma \leqq t \leqq \tau
$$

and

$$
\left(t, z_{0}(t), z_{0}^{\prime}(t)\right) \in D \quad \text { for } \quad \sigma \leqq t \leqq \tau
$$

since $D_{2}$ and $D_{4} \cup D_{5}$ are negatively invariant set of (3.12). Therefore the 
points $q_{y}$ and $q_{z}$ belong to $H$. From this and the fact that $q$ is also a left end point of $H \cap L$, we have $H \cap S_{0}$ is nonempty. This contradicts $H \subset K$ and $K \cap S_{0}=\varnothing$. Thus we have $K \cap S_{0}$ is nonempty, which assures the existence of a solution of (1.1) joining a point of $S_{0}$ and a point of $E$.

q.e.d.

REMARK. As will be seen in the following example, in Theorem 3.1, we cannot arbitrarily choose the value of $x(a)$ in $\underline{\omega}(a) \leqq x(a) \leqq \bar{\omega}(a)$. Consider the equation

$$
x^{\prime \prime}=0
$$

for $0 \leqq t \leqq 1$. Let $\underline{\omega}, \bar{\omega}, \underline{\Omega}$ and $\bar{\Omega}$ be constant functions such that

$$
\underline{\omega}(t)=-1, \quad \bar{\omega}(t)=1, \underline{\Omega}(t, x)=-1 \text { and } \bar{\Omega}(t, x)=1 .
$$

Though all conditions in Theorem 3.1 are satisfied, there is no solution $x(t)$ of (3.13) satisfying $x(0)=-1$ and $x(1)=1$ in the domain $D$.

CoROLLARY 3.1. Let $f(t, x, y)$ be a continuous function on a domain $W:-\infty \leqq a<t<b<\infty, \underline{\omega}(t) \leqq x \leqq \bar{\omega}(t), \underline{\Omega}(t, x) \leqq y \leqq \bar{\Omega}(t, x)$, where $\underline{\omega}, \bar{\omega}, \underline{\Omega}$ and $\bar{\Omega}$ are those in Theorem 3.1 and satisfy inequalities (3.4), (3.5) and (3.6) replacing $a \leqq t \leqq b$ by $a<t<b$. Furthermore, assume that there is a number $T, a<T<b$, and a measurable function $m(t)$ on $T \leqq t<b$ and a Lebesgue integrable function $h(t)$ on $T \leqq t<b$ such that

$$
\begin{gathered}
|f(t, x, y)| \leqq m(t) \\
\text { for } \quad T \leqq t<b, \quad \underline{\omega}(t) \leqq x \leqq \bar{\omega}(t), \quad \underline{\Omega}(t, x) \leqq y \leqq \bar{\Omega}(t, x)
\end{gathered}
$$

and

$$
\int_{T}^{t} m(s) d s \leqq h(t) \quad \text { for } \quad T \leqq t<b .
$$

Then the functions $\underline{\omega}(t)$ and $\bar{\omega}(t)$ are necessarily bounded on $T \leqq t<b$, and for any number $x_{b}$ such that

$$
\liminf _{t \rightarrow b} \underline{\omega}(t) \leqq x_{b} \leqq \limsup _{t \rightarrow b} \bar{\omega}(t),
$$

equation (1.1) has at least one solution $x(t)$ defined on the whole interval $(a, b)$ satisfying $x\left(b^{-}\right)=\lim _{t \mid b} x(t)=x_{b}$.

Proof. We can choose two sequences $\left\{a_{n}\right\}$ and $\left\{b_{n}\right\}$ such that

$$
\begin{aligned}
& a<a_{n+1}<a_{n}<T, \quad(n=1,2, \cdots), \lim _{n \rightarrow \infty} a_{n}=a, \\
& b>b_{n+1}>b_{n}>T, \quad(n=1,2, \cdots), \quad \lim _{n \rightarrow \infty} b_{n}=b
\end{aligned}
$$


and

$$
\limsup _{n \rightarrow \infty} \underline{\omega}\left(b_{n}\right) \leqq x_{b} \leqq \liminf _{n \rightarrow \infty} \bar{\omega}\left(b_{n}\right) \text {. }
$$

Let $W_{n}(n=1,2, \cdots)$ be a compact domain defined by

$$
a_{n} \leqq t \leqq b_{n}, \quad \underline{\omega}(t) \leqq x \leqq \bar{\omega}(t), \quad \underline{\Omega}(t, x) \leqq y \leqq \bar{\Omega}(t, x) .
$$

It then follows from Theorem 3.1 and (3.15) that there exists a solution $x_{n}(t)$ of (1.1) defined on $\left[a_{n}, b_{n}\right]$ such that

$$
x_{n}\left(b_{n}\right)=B_{n} \rightarrow x_{b} \quad \text { as } n \rightarrow \infty \text {. }
$$

By standard arguments, we may assume that there is a solution $x(t)$ of (1.1) defined on $(a, b)$ such that

$$
x_{n}(t) \rightarrow x(t) \text { and } x_{n}^{\prime}(t) \rightarrow x^{\prime}(t)
$$

uniformly on any compact interval in $(a, b)$, as $n \rightarrow \infty$. For any fixed $t, T \leqq t<b$, we may assume $t<b_{n}(n=1,2, \cdots)$. Since $x_{n}(t)$ is a solution of (1.1) on $\left[a_{n}, b_{n}\right]$, we can write

$$
\begin{aligned}
B_{n}= & x_{n}\left(b_{n}\right)=x_{n}(t)+\int_{t}^{b_{n}} x_{n}^{\prime}(s) d s \\
= & x_{n}(t)+\int_{t}^{b_{n}}\left\{x_{n}^{\prime}(T)+\int_{T}^{s} x_{n}^{\prime \prime}(u) d u\right\} d s \\
= & x_{n}(t)+\left(b_{n}-t\right) x_{n}^{\prime}(T) \\
& \quad+\int_{t}^{b_{n}} \int_{T}^{s} f\left(u, x_{n}(u), x_{n}^{\prime}(u)\right) d u d s,
\end{aligned}
$$

which implies

$$
B_{n}=x_{n}(t)+\left(b_{n}-t\right) x_{n}^{\prime}(T)+\int_{t}^{b} F_{n}(s) d s
$$

where

$$
F_{n}(s)= \begin{cases}\int_{T}^{s} f\left(u, x_{n}(u), x_{n}^{\prime}(u)\right) d u & \text { for } T \leqq s \leqq b_{n} \\ 0 & \text { for } b_{n}<s<b .\end{cases}
$$

From the assumptions, we have

$$
\begin{gathered}
\left|F_{n}(s)\right| \leqq \int_{T}^{s}\left|f\left(u, x_{n}(u), x_{n}^{\prime}(u)\right)\right| d u \leqq \int_{T}^{s} m(u) d u \leqq h(s) \\
\text { for } T \leqq s<b .
\end{gathered}
$$

Therefore, by setting $t=T$ in (3.16), $\left|B_{n}\right|<M$ for some constant $M$ independent of $n$. This implies that $x_{b}$ is a finite number, and hence $\underline{\omega}(t)$ and $\bar{\omega}(t)$ are bounded on $T \leqq t<b$ because $x_{b}$ is an arbitrary 
number satisfying (3.14). On the other hand,

$$
\lim _{n \rightarrow \infty} F_{n}(s)=\lim _{n \rightarrow \infty} \int_{T}^{s} x_{n}^{\prime \prime}(u) d u=\lim _{n \rightarrow \infty}\left(x_{n}^{\prime}(s)-x_{n}^{\prime}(T)\right),
$$

that is

$$
\lim _{n \rightarrow \infty} F_{n}(s)=x^{\prime}(s)-x^{\prime}(T) \quad \text { for } \quad T \leqq s<b .
$$

Hence $x^{\prime}(s)$ is Lebesgue integrable function on $T \leqq s<b$ by (3.17). It follows from (3.16), (3.17) and (3.18) that

$$
\begin{aligned}
x_{b} & =x(t)+(b-t) x^{\prime}(T)+\int_{t}^{b}\left(x^{\prime}(s)-x^{\prime}(T)\right) d s \\
& =x(t)+\int_{t}^{b} x^{\prime}(s) d s \quad \text { for } \quad T \leqq t<b .
\end{aligned}
$$

Since $x^{\prime}(s)$ is Lebesgue integrable on $T \leqq s<b$, we have

$$
\int_{t}^{b} x^{\prime}(s) d s \rightarrow 0 \quad \text { as } t \rightarrow b^{-},
$$

which implies $x\left(b^{-}\right)=x_{b}$.

q.e.d.

4. Appendix. When $B$ is locally connected, the assumption that $B^{-}$ is open in $B$ cannot be dropped in Theorem 2.1. This is observed in the following proposition.

Proposition 4.1. Let $\mathscr{F}$ be a family of curves and each point of $D=D(\mathscr{F})$ be a left Kneser point and $B=\partial D$ be locally connected. If the set

$$
K(E)=Z^{-}(E) \cap\left(B^{l} \cup B_{-}\right)
$$

is a connected set for any continuum $E$ in $D$, then $B^{-}$is open in $B$. Therefore $K(E)$ is necessarily continuum.

Proof. Assume $B^{-}$is not open in $B$. Then there is a point $p$ in $B^{-}$which is an accumulation point of $B^{l} \cup B_{-}$. Since $p \in B^{-}$, we have $p$ does not belong to $\overline{K(p)}$. Therefore there is a positive number $\varepsilon$ such that

$$
V_{\varepsilon}(p) \cap K(p)=\varnothing,
$$

where $V_{\varepsilon}(p)$ is an open $\varepsilon$-neighborhood of $p$ in $B$. Let $\delta, 0<\delta<\varepsilon$, be arbitrary. By the local connectedness of $B$, there is a neighborhood base $\left\{U_{n}: n=1,2, \cdots\right\}$ of $p$ in $B$ consisting of continua such that

$$
U_{n} \subset V_{\hat{o}}(p) \quad(n=1,2, \cdots) .
$$


For each $n$, there is a point $r_{n}$ in $U_{n} \cap\left(B^{l} \cup B_{-}\right)$since $p$ is an accumulation point of $B^{l} \cup B_{-}$. Namely, the connected set $K\left(U_{n}\right)$ contains an interior point $r_{n}$ of $V_{\delta}(p)$ and exterior part $K(p)$ of $V_{\delta}(p)$, and hence $K\left(U_{n}\right) \cap \partial V_{\delta}(p)$ is nonempty, where $\partial V_{\delta}(p)$ is the boundary of $V_{\delta}(p)$ in $B$. Thus there exist $p_{n} \in U_{n}, q_{n} \in \partial V_{\delta}(p)$ and $x_{n} \in \mathscr{F}$ whose right end point is $p_{n}$ and left end point is $q_{n}$. By the compactness of $\mathscr{F}$, we may assume that there is a curve $x \in \mathscr{F}$ to which $x_{n}$ converges. Obviously, the right end point of $x$ is $p$, and the left end point of $x$ denoted by $q$ belongs to $\partial V_{\delta}(p)$, and hence $\operatorname{dist}(p, q)=\delta$. Since $\delta>0$ be arbitrarily small, we have $p \in B_{-}$. This contradicts the assumption $p \in B^{-}$.

q.e.d.

\section{REFERENCES}

[1] M. HukuHARA, Familles knesériennes et le problème aux limites pour l'équation différentielle ordinaire du second ordre, I, Publ. RIMS, Kyoto Univ. Ser. A, 3 (1967), $243-270$.

[2] P. Hartman, Ordinary Differential Equations, John Wiley and Sons, Inc. 1964.

Mathematical Institute

TôHOKU UNIVERSITY

SENDAI, JAPAN 
